To appear in the International Journal of Remote Sensing

Vol. 00, No. 00, Month 20XX, 1-17

\title{
A dual-polarimetric approach to earthquake damage assessment
}

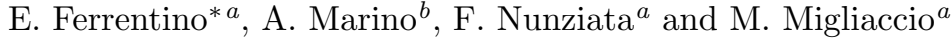 \\ ${ }^{a}$ Università degli Studi di Napoli Parthenope, \\ Dipartimento di Ingegneria, Napoli, Italy \\ ${ }^{b}$ The University of Stirling, \\ Natural Sciences, Stirling, United Kingdom \\ (Received 00 Month 20XX; accepted 00 Month 20XX)
}

\begin{abstract}
In this study, a novel physical approach is proposed to detect damages due to earthquakes using dual polarimetric (DP) coherent Synthetic Aperture Radar (SAR) imagery. An optimisation method, aimed at enhancing scattering basis differences between measurements collected before and after the event, is designed exploiting Lagrange optimisation of the difference between two polarimetric covariance matrices. A meaningful showcase is presented to demonstrate the soundness of the proposed approach that consists of processing Sentinel-1 C-band scenes related to 2016 Central Italy Earthquake. The proposed approach, which is contrasted with the conventional coherence based single- and dual-polarisation approaches, results in the best sensitivity to damages.
\end{abstract}

Keywords: SAR, polarimetry, earthquake, damage assessment

\section{Introduction}

Earthquakes are tremendous natural catastrophes that can not still predicted with current technology and knowledge. In the past decade, several earthquakes around the world killed hundreds of thousands of people. After earthquakes, a fast damage assessment is of crucial importance to support effective emergency response actions and hence, to avoid or reduce the impact of the disaster. Among the remote sensing tools, the Synthetic Aperture Radar (SAR), due to its all-day and its

\footnotetext{
*Corresponding author. Email: emanuele.ferrentino@uniparthenope.it
} 
almost all-weather fine spatial resolution imaging capabilities, can be very useful for earthquake monitoring. Typically, pre- and post-event single-polarization SAR imagery are exploited to design change detection methods (Cihlar, Pultz, and Gray 1992; $\mathrm{Hu}$ and Ban 2014; Marin, Bovolo, and Bruzzone 2015; Rignot and van Zyl 1993). The single-polarisation features adopted in most of the cases are the interferometric coherence and the amplitude of the backscattered signal Yonezawa and Takeuchi 2001; Stramondo et al. 2006; Matsuoka and Yamazaki 2004). Very often, those approaches are augmented using external auxiliary information, e.g.; optical imagery. A key drawback of the approaches based on the interferometric coherence relies on the fact that they generally overestimate earthquakes damages due to the temporal decomposition that intrinsically affects the pre- and post-event acquisitions. In (Gong et al. 2016), very high resolution (VHR) TerraSAR-X imagery and original building footprint maps are exploited to identify buildings destroyed by earthquakes. Finally, each building is classified into damage classes using machine learning techniques, i.e.; a non-physical approach. Authors claim that, in about $90 \%$ of the cases, the method is able to distinguish between collapsed and standing buildings. In (Gamba, Dell'Acqua, and Lisini 2006) an approach that combines pixel-based and feature-based techniques is proposed to detect changes in urban areas using airborne SAR (AIRSAR) and satellite ENVISAT Advanced SAR (ASAR) images. Experimental results show an improvement in the change maps using both pixel- and feature-information. Moreover, the technique is able to overcome major problems arising from multi-temporal image analysis, e.g.; misregistration or miscalibration.

Indeed, when two SAR acquisitions must be analyzed, since the related acquisition geometries are never identical, even if the same sensor is exploited, the preliminary step of coregistration must be accomplished. This step can benefit of the band-limited nature of the SAR system; however, it introduces coherence losses (Migliaccio et al. 2007). Further, the use of two multitemporal SAR acquisitions call for an accurate and stable measurement process over time that can be of special challenge when a SAR constellation is to be exploited.

Recently, a large number of SAR satellite missions equipped with polarimetric SARs have been launched. The availability of polarimetric SAR measurements triggered the development of added-value products in different thematic domains, including land applications Lee and Pottier (2009). Within this context, several 
studies have been proposed to exploit full-polarimetric (FP) SAR measurements to detect damages due to earthquake (Watanabe et al. 2012; Sato, Chen, and Satake 2012, Chen and Sato 2013; Park, Yamaguchi, and jin Kim 2013; Chen, Wang, and Sato 2016 ?). Although FP SAR offers the largest amount of information about the observed scene, hardware and budget considerations suggest the operational use of simpler polarimetric configurations, e.g.; the DP SAR (Nunziata, Migliaccio, and Li 2015: Buono et al. 2016). In (Watanabe et al. 2016; Karimzadeh and Mastuoka 2017) DP SAR data are exploited for earthquake observation. These studies deal with the discrimination between the collapsed and preserved buildings that is a key information to perform damaged areas detection. Both intensity and coherence features, evaluated using DP L- and C-band SAR imagery, are exploited to show that cross-polarized channel can help identifying collapsed building. In Ferrentino et al. 2018), a new mehod that exploits DP SAR measurements to detect damages due to earthquakes is proposed. The method is based on the reflection symmetry property that, measured using the inter-channel complex coherence, is shown to perform better than SP and DP amplitude ones. The above-mentioned studies exploit only part of the information carried on the covariance matrix, i.e.; interchannel-correlation, squared modulus of the channels, etc. To the best of our knowledge, no study relies on the exploitation of the complete covariance matrix. In this study, a new paradigm is proposed to detect damages due to earthquakes in DP SAR imagery. The paradigm consists of optimising the difference between two covariance matrices (collected before and after the earthquake, respectively) through a Lagrange optimisation method. This results in an eigenvalues problem whose eigenvalues are shown to provide remarkable sensitivity to earthquake-induced changes. Once the eigenvalues are obtained, an unsupervised approach is designed to generate binary imagery where changes are easily discernible. A meaningful showcase is presented to discuss the performance of the proposed methodology that consists of processing DP SAR scenes collected by the Copernicus Sentinel-1 mission over the area affected by the Central Italy earthquake occurred in 2016 . The obtained results are visually contrasted with both optical imagery and results obtained by independent studies. The comparison confirms the soundness of the proposed methodology that succeeds in detecting damaged areas. In addition, the new method is also contrasted with state-of-the-arts SP and DP methods and it is shown to result in the best sensitivity to damaged areas. 


\section{Methodology}

A full-polarimetric (FP) SAR measures, for each resolution cell, the scattering matrix $\mathbf{S}$, i.e.; a $2 \times 2$ matrix of complex elements, termed as scattering amplitudes. In the backscattering case and under the assumption of reciprocity, $\mathbf{S}$ is given by Cloude 2009; Lee and Pottier 2009)

$$
\mathbf{S}=\left[\begin{array}{ll}
S_{\mathrm{HH}} & S_{\mathrm{HV}} \\
S_{\mathrm{HV}} & S_{\mathrm{VV}}
\end{array}\right]
$$

where a linear horizontal $(H)$-vertical $(V)$ orthogonal basis is adopted. The scattering matrix rules the transformation of the incident electromagnetic wave into the scattered one according to the Jones formalism. In practical cases, hardware and budget constraints may suggest a simpler polarimetric SAR that measures only a subset of the scattering matrix. This is the case, for instance, of a DP SAR that measures only the first or the second row of $\mathbf{S}$ (1) or a single-polarimetric SAR that measures only one term of $\mathbf{S}$.

The scattering matrix (1) of one single pixel is only able to describe a fully polarised target and it is not able to take into account depolarization. In order to overcome such drawback, second-order descriptors must be employed (Cloude 2009, Lee and Pottier 2009). Hence, a coherent approach, based on the construction of the covariance matrix $\mathbf{C}$, can be adopted. $\mathbf{C}$ is a semi-positive definite (PSD) Hermitian matrix:

$$
\mathbf{C}=\left\langle\underline{\boldsymbol{k}}_{\mathrm{L}} \cdot \underline{\boldsymbol{k}}_{\mathrm{L}}^{* \mathrm{~T}}\right\rangle
$$

where $\langle\cdot\rangle$ is the finite averaging opearator and $\underline{\boldsymbol{k}}_{\mathrm{L}}=\left[S_{x x}, S_{x y}\right]^{\mathrm{T}}$, is the scattering vector, i.e.; the projection of $\mathbf{S}$ according to the lexicographic basis Cloude 2009, Lee and Pottier 2009). Since $\mathbf{C}$ is a PSD Hermitian matrix, it can be uniquely decomposed according to the eigenvalue/eigenvector decomposition Cloude and Pottier 1997):

$$
\mathbf{C}=\mathbf{U D U}^{\mathrm{T}}
$$


where $\mathbf{U}$ and $\mathbf{U}^{\mathrm{T}}$ are unitary orthogonal matrices, T stands for transpose and $\mathbf{D}$ is a diagonal matrix whose elements are the real and non negative eigenvalues of $\mathbf{C}$. In the FP case, the decomposition (3) allows interpreting the general scattering targets described by $\mathbf{C}$ in terms of three elementary well-known mechanisms (Cloude 2009; Lee and Pottier 2009). In the DP case, the decomposition (3) can be still applied but the physical interpretation may not be straightforward (Cloude 2007; Ainsworth et al. 2008).

In this study, a different approach is proposed that consists of analysing the "change matrix" $\mathbf{C}_{\mathrm{CD}}$ that is defined as follows:

$$
\mathbf{C}_{\mathrm{CD}}=\mathbf{C}_{2}-\mathbf{C}_{1}
$$

where $\mathbf{C}_{1}$ and $\mathbf{C}_{2}$ are the covariance matrices that characterize DP SAR acquisitions collected with the same geometry over the same scene but at two different times. We refer to $\mathbf{C}_{\mathrm{CD}}$ as the change matrix. $\mathbf{C}_{\mathrm{CD}}$ is not Hermitian, however its diagonal elements are real and the upper triangular part is the complex conjugate of the lower triangular part (Marino and Hajnsek 2014). This implies that, unlike $\mathbf{C}, \mathbf{C}_{\mathrm{CD}}$ is not bounded to be PSD; hence, its quadratic form:

$$
P=\underline{\omega}^{* \mathrm{~T}} \mathbf{C}_{\mathrm{CD}} \underline{\omega} \in \Re
$$

where $\underline{\omega}=\frac{\mathbf{k}}{|\mathbf{k}|}$ is the projection scattering vector, can be negative. The possibility to have negative values implies that it can take into account decreases or increases of a given scattering mechanism. The trace:

$$
\operatorname{Trace}\left(\mathbf{C}_{\mathrm{CD}}\right)=\lambda_{1 \mathrm{CD}}+\lambda_{2 \mathrm{CD}}
$$

where $\lambda_{1 \mathrm{CD}}$ and $\lambda_{2 \mathrm{CD}}$ are the two eigenvalues associated to the matrix $\mathbf{C}_{\mathrm{CD}}$, takes into account the overall power associated to the changes in the partial target, and it also can be either positive or negative. These features are interesting from a physical viewpoint since they make $\mathbf{C}_{\mathrm{CD}}$ sensitive to changes in scattering mechanisms (5) as well as their overall power (6).

By optimizing $\mathbf{C}_{\mathrm{CD}}$ over all the possible projection vectors $\underline{\omega}$, it is possible to derive the projection vectors that exhibit the largest or smallest changes, i.e.; the 
projection vectors that maximize/minimize changes (Marino and Alonso-Gonzlez 2017). From a mathematical viewpoint, this can be undertaken optimising the quadratic form $P$ :

$$
\underline{\omega}_{\max }=\underset{\underline{\omega} \in \mathbb{C}^{3}}{\operatorname{Argmax}}\left[\underline{\omega}^{* \mathrm{~T}} \mathbf{C}_{\mathrm{CD}} \underline{\omega}\right]
$$

with $\omega$ being a unitary matrix. The well-known Lagrange optimization method (Lee and Pottier 2009; Cloude 2009, Marino and Hajnsek 2014; Marino and AlonsoGonzlez 2017) can be applied that leads to the Lagrangian function:

$$
L=\underline{\omega}^{* \mathrm{~T}} \mathbf{C}_{\mathrm{CD}} \underline{\omega}-\lambda\left(\underline{\omega}^{* \mathrm{~T}} \underline{\omega}+\mathbf{C}\right)
$$

Differentiating over $\partial \underline{\omega}^{* T}$ and setting the derivative equal to zero

$$
\frac{\partial L}{\partial \underline{\omega}^{* T}}=\mathbf{C}_{\mathrm{CD}} \underline{\omega}-\lambda \underline{\omega}=0
$$

we obtain the equation:

$$
\mathbf{C}_{\mathrm{CD}} \underline{\omega}=\lambda \underline{\omega}
$$

This is an eigenvalue problem that provides the eigenvalues $\lambda_{i}$ and the eigenvectors $\underline{u}_{i}$ (with $i$ going from 1 to 2 ) of $\mathbf{C}_{\mathrm{CD}}$ that maximize/minimize the power difference between $\mathbf{C}_{11}$ and $\mathbf{C}_{22}$. We decided to focus on the power difference since it best suits to additive changes in terms of scattering mechanism. In fact, we expect that changes resulting after the earthquake may either introduce a new scattering mechanism or remove an existing one.

In this study, the sum of the absolute value of the two eigenvalues is considered as an indicator of the changes occurred between the two SAR acquisitions in the same scene:

$$
\lambda_{\text {tot }}=\left|\lambda_{1}\right|+\left|\lambda_{2}\right|
$$

In fact, the largest eigenvalue $\lambda_{1}$ of the matrix $\mathbf{C}_{\mathrm{CD}}$ is mainly positive, which 
means that it represents the power of scattering contributions that increase or appear in the second acquisition. The smallest eigenvalue $\lambda_{2}$ is mostly negative, which means that it represents the power of scattering mechanisms that are reduced or disappear when the scene is observed in the second acquisition (Marino and Hajnsek 2014; Marino and Alonso-Gonzlez 2017). To analyse the sensitivity of the detector to damages due to the earthquake, two cases are addressed: a) the two imagery are both acquired before the seismic event. In this case $\lambda$ is termed with the subscript "pre"; b) the two imagery are acquired before and after the seismic event. In this case, $\lambda$ is termed with the subscript "int".

To fully appreciate the extra-information resulting from the exploitation of the complete DP covariance matrix, a single-polarization feature is considered for reference purposes. This feature, is based on the element $\mathbf{C}_{x x}$ of the covariance matrix (2), where $x$ can be either 1 or 2 , and consists of undertaking the following difference $\Delta$ :

$$
\Delta=\mathbf{C}_{x x}^{p}-\mathbf{C}_{x x}^{j}
$$

where $p$ and $j$ refer to two SAR scenes collected: $a$ ) both before the earthquake. In this case $\Delta$ is termed as $\left.\Delta_{\text {pre }} ; b\right)$ before $(p)$ and after $(j)$ the earthquake. In this case $\Delta$ is termed as $\Delta_{i n t}$. The index $\Delta_{\text {int }}$ is expected to provide information on the changes occurred after the earthquake. In fact, the scenario is expected to change after the earthquake due, for instance, to collapsed building. Those phenomena result in changes in the backscattered signal resulting from the pre- and postevent imagery that can be used to detect the changes due to the earthquake. In addition, to analyse the sensitivity of co- and cross-polarized channels to damaged areas, $\Delta, 12$ is evaluated using $\mathbf{C}_{11}\left(\Delta_{\text {co }}\right)$ or $\mathbf{C}_{22}\left(\Delta_{\mathrm{xc}}\right)$. In fact, co- and crosspolarized channels are inherently linked to different scattering mechanisms, hence it is worth expecting that $\Delta_{\mathrm{co}}$ and $\Delta_{\mathrm{xc}}$ carry different scattering-based information that can be jointly used to provide a deeper understanding of the damaged area.

\subsection{Unsupervised change detector}

In this subsection the unsupervised change detector that allows obtaining a binary imagery where changes are clearly highlighted is described. The method is based on the cell-averaging constant false alarm rate (CA-CFAR) (Barboy, Lomes, and 
Perkalski 1986) that is applied to both $\lambda$ and $\Delta$ imagery. The CA-CFAR method has two advantages: a) it allows not using guard windows and therefore avoid problems with contamination or size of the target, b) it allows rejecting some of the areas that are by their nature highly affected by changes (e.g. agricultural fields), this is because the detectors will have large values and therefore set high thresholds on those targets.

A key step to design a CFAR detector relies on the analysis of the statistic of the clutter. In this study, to evaluate the statistics of $\lambda$ and $\Delta$ over the clutter, a region of interest (ROI) excerpted outside the city of Amatrice (see the white box in Figure 22 is considered. Note that, the considered ROI is related to an area where no changes occurred. This means that the statistic is the same in the pre-and interseismic case. The empirical probability density function (pdf) of $\Delta$ and $\lambda$ are shown in Figure 3 where an exponential pdf and a Lognormal pdf are also depicted as reference distributions for $\Delta$ and $\lambda$, respectively. The Kolmogorov-Smirnov binary hypothesis test, undertaken using a significance level equal to 0.05 , confirms that the Lognormal and the Exponential models well approximate the distribution of $\Delta$ and $\lambda$ background clutter, respectively. Note that, in this study, clutter is meant as all the areas that are not urbanized. Once approximate pdfs are found for $\Delta$ and $\lambda$ over the clutter, the relationship between Probability of false alarm $\left(P_{\mathrm{fa}}\right)$ and the threshold (th) can be provided. When dealing with $\Delta$, the relationship between th and $P_{\mathrm{fa}}$ is given by (Buono et al. 2014):

$$
P_{\mathrm{fa}}=\int_{\mathrm{th}}^{\infty} \gamma e^{-\gamma x} \mathrm{dx}
$$

where the threshold is given by:

$$
\text { th }=-\frac{1}{\gamma} \ln \left(P_{\mathrm{fa}}\right)
$$

with $\gamma$ being the distribution rate parameter value of the exponential distribution.

When dealing with $\lambda$, the relationship between the detection threshold th and 
the probability of false alarm $P_{\mathrm{fa}}$ is given by (Sayama and Ishii 2014):

$$
P_{\mathrm{fa}}=\int_{\mathrm{th}}^{\infty} \frac{1}{x \sigma \sqrt{2 \pi}} e^{-\frac{(\ln (x)-\mu)^{2}}{2 \sigma^{2}}} \mathrm{dx}
$$

where the threshold is given by:

$$
\operatorname{th}=e^{\sqrt{2} \sigma \operatorname{erf}^{-1}\left(1-2 P_{\mathrm{fa}}\right)}
$$

where $\mu$ and $\sigma$ are the mean and standard deviation values of the Lognormal distribution, respectively and $\operatorname{erf}(\cdot)$ stands for the erf function.

\section{Experiments}

In this section we present and discuss the new multi-polarization approaches to detect earthquake damages.

The satellite dataset consists of eight single look complex (SLC) DP SAR imagery collected before and after the 2016 Centre Italy earthquake by the Copernicus Sentinel-1 mission over the area of Amatrice (Lazio, Italy) in ascending and descending orbits, with an incident angle around $42^{\circ}$ (see Table 1 ).

The study case refers to the area damaged by the earthquake that, on August 24, 2016, hit a large portion of the central Apennines fold and thrust belt, between the towns of Norcia and Amatrice. Seismological data, collected by the Istituto Nazionale di Geofisica e Vulcanologia (INGV) seismic network, show that the area mostly affected by the earthquake extended for about $40 \mathrm{~km}$ along with the NWSE direction. The most affected area included the towns of Norcia and Amatrice and the event resulted in nearly 300 fatalities, thousands of wounded and over 3000 displaced people (Albano et al. 2016). In Figure 1, Google Earth ${ }^{\circledR}$ images showing the city of Amatrice before (Figure 1 $(a)$ ) and after (Figure 1 $(b)$ ) the earthquake are provided. The ground-projected excerpts of the SAR imagery collected over the area of Amatrice before and after the earthquake are shown in Figure 2, see the first and the second columns, respectively. The VV-polarized scenes collected in ascending and descending mode are shown in the first and the second row, respectively. To discuss the role played by the SAR pass, experiments are organized into two part. The first one deals with SAR imagery collected in ascending pass; 
the second one refers to SAR scenes collected over the same area in descending pass.

The first experiment consists of processing DP SAR scenes collected in ascending mode, see Figure 2 where squared modulus VV SAR scenes collected on 22 August 2016 ( $a$ ) and 3 September 2016 (b), i.e.; before and after the earthquake, respectively, are shown in the first row.

First, the SP method described in Section 2 is applied to the pre- and postevent SAR imagery collected using the $\mathrm{VV}$ and $\mathrm{VH}$ polarizations to generate $\Delta_{\text {co }}$ and $\Delta_{\mathrm{xc}}$, see Figure $4(a)$ and $(b)$, respectively. Note that changes associated to damages due to the earthquake are visible both in the co-polarized image (Figure $4(a))$ and in the cross-polarized one (Figure $4(b)$ ). Note that different scales are adopted in Figure $4(a)$ and $4(b)$. However changes are better highlighted by $\Delta_{\text {co }}$ that shows significant differences between the pre- and post-event imagery within the area of the Amatrice city. In particular, the largest changes apply in the upper part of the city of Amatrice. This agrees with both Google Earth ${ }^{\complement}$ image (shown in Figure 1) and the study carried out by INGV (Romaniello et al. 2016) that indicates the upper part of Amatrice as the area most affected by damages. To test the robustness of the approach against changes related to the earthquake, we applied the method to a couple of SAR imagery collected before the earthquake. Both $\Delta_{\text {co }}$ and $\Delta_{\text {xc }}$ imagery (not shown to save space) result in negligible differences within the area of Amatrice.

The differences shown by co- and cross-polarized channels suggest to exploit those channels in a joint way to maximise the sensitivity to damaged areas. Hence, the full covariance matrix is exploited using the approach described in Section 2 that consists of evaluating the eigenvalues $\lambda_{i}$ of the difference between covariance matrices that refer to two DP SAR scenes collected before the earthquake and during the inter-seismic stage of the earthquake $\left(\mathbf{C}_{\mathrm{CD}}\right)$. In Figure 5 , the false color imagery associated to the two eigenvalues $\lambda_{1}$ and $\lambda_{2}$ and to the metric (11) are shown in Figure $5(a),(b)$ and $(c)$, respectively. The urban areas that correspond to the city of Amatrice is circled. It can be noted that $\lambda_{1}, \lambda_{2}$ and $\lambda_{\text {tot }}$ imagery clearly witness that changes occurred. It can be noted that, as expected, $\lambda_{1}$ is generally positive; while $\lambda_{2}$ is mostly negative. In addition, $\lambda_{1}$ and $\lambda_{2}$ provide almost complementary information that are jointly exploited to maximize the changes when $\lambda_{\text {tot }}$ is used, see Figure $5(c)$. Note that, even in this case, to test the robustness of the 
proposed method, a couple of SAR imagery both collected before the seismic event is processed. Results (not show to save space) point out that negligible changes apply.

A visual comparison between the SP method $\left(\Delta_{\text {co }}\right.$, see Figure $\left.4(a)\right)$ and the DP method $\left(\lambda_{\text {tot }}\right.$, see Figure $5(c)$ ) shows that the latter improves the detection of damages, in particular in the upper part of the city of Amatrice, i.e.; the area most affected by damages. A deeper analysis on $\lambda_{1}$ and $\lambda_{2}$ imagery shows that they carry on information related to both co- and cross-polarized channels. In fact, $\lambda_{1}$ (Figure $5(a)$ ) provides results that are very close to $\Delta_{\text {co }}$ (Figure 4 (a); while $\lambda_{2}$ (Figure $5(b)$ ) exhibits values that are significantly different from $\Delta_{\mathrm{xc}}$ (Figure 4 (b)) witnessing that it results from the combination of both co- and cross-polarized channels. Hence, the use of the full covariance matrix through $\lambda_{1}, \lambda_{2}$ and $\lambda_{\text {tot }}$ (11) results in the best detection of changes associated to the earthquake.

Note that changes are detected also out of the area of Amatrice. Most of them are actually related to damages occurred after the earthquake. In particular, focusing on changes that are visible on the rightmost part of Figure 5 (see green and yellow rectangles overlaid on Figure $5(c)$ ), one can note that they are actually related to damages occurred after the earthquake that hit the city of Castel Sant'Angelo, Rieti (green box) and urban area of Sommati, Rieti (yellow box). Those cities where significantly affected by the earthquake as one can noted by looking Google Earth $^{\complement}$ imagery collected before and after the Earthquake, see Figure 6 and 7.

To fully appreciate the advantages of exploiting coherently the two channels (i.e.; both the amplitude an their phase), results of the DP metric $\lambda_{\text {tot }}$ are inter-compared with results obtained using an incoherent combination of co- and cross-polarized channel. The incoherent metric used in this study is the difference between the $S P A N$, i.e.; the sum of squared modulus of the channels, evaluated using a pair of images acquired before and after the earthquake. The result is shown in Figure 8 . It can be noted that $S P A N$ is able to detect the changes occurred after the seismic event. However, the DP metric (Figure $5(c)$ ) results in a better detection of the changes in particular in the upper part of the Amatrice city.

To provide a binary output that highlights changes associated with the earthquake, the CA-CFAR (see Section 2.1) is adopted. According to Equations (14) and 16), th is predicted for $\Delta$ and $\lambda$ using $P_{\mathrm{fa}}$ equal to $10^{-5}$ that resulted in the best compromise between detection and false alarms. The Exponential and Lognormal 
distribution parameters are obtained using the "pre" imagery filtered with a $N \times N$ moving window with $\mathrm{N}$ equal to 100 . The CA-CFAR method is applied to both the pre-event and inter-seismic event cases (see the second and the first columns, respectively). The binary imagery show in Figure 9 are arranged according to the matrix format: the first column refers to the interseismic case; while the second column refers to the pre-event case. The three rows refer to $\Delta_{\text {co }}, \Delta_{\mathrm{xc}}$ and $\lambda_{\text {tot }}$, respectively. By visually inspecting binary imagery of Figure 9 one can note that: when dealing with the inter-seismic case, all the features result in non-negligible changes. This witness that changes associated to the earthquake are detected. The best detection performance is provided by $\lambda_{\text {tot }}$ (Figure 9 $(e)$ ); while only some hints of signals are visible in $\Delta_{\mathrm{xc}}$ image (Figure 9 (c)). When dealing with the inter-seismic case, all the features result in non-negligible changes witnessing the robustness of all the features with respect to variability of the environment. Even in this case, one can note that the extra-benefit of the cross-polarized channel can be only exploited coherently combining co- and cross-polarized channels, i.e.; by exploiting the whole covariance matrix. To quantitatively assess the improvement of the DP method, pixels inside the red ellipse in Figure 9 are summed up. Results, listed in Table 2, show that $\lambda_{\text {tot }}$ exhibits $44 \%$ pixels more than $\Delta_{\text {co }}$ and $92 \%$ pixels more than $\Delta_{\mathrm{xc}}$. Although a detailed ground truth is not available, previous researches focused on the same area (Romaniello et al. 2016) and Google Earth $^{\complement}$ imagery confirm that the extra-changes detected by $\lambda_{\text {tot }}$ are likely due to the earthquake since they are located in the upper-part of the Amatrice city. The second experiment consists of processing DP SAR scenes collected in descending mode, see Figure 2 where squared modulus VV SAR scenes collected on 21 August $2016(c)$ and 2 September $2016(d)$ are shown in the second row. Results obtained using both SP and DP methods, see Figure 10 and 11, respectively, are similar to the results obtained from the first experiment. Note that descending datasets are acquired with a difference of one day with respect to the ascending datasets. This means that the differences between the two datasets are mainly due to the different acquisition geometry. Once again, the DP method results in the largest number of change pixels over the urban area of Amatrice. Even in this case, changes are detected also out of the area of Amatrice. In particular, the same two areas of the first experiment are considered (see green and yellow boxes). Note that, due to the different acquisition geometry of the datasets, these areas are located in 
different places with respect to the ascending pass imagery. Even in this case, both SP and DP methods are able to detect the changes occurred. The binary imagery, obtained using the CA-CFAR approach with the same parameters of the previous experiment, are shown in Figure 12. To quantitatively assess the improvement of the DP method, once again pixels inside the red ellipse in Figure 12 are summed up. Results, listed in Table 2, show that $\lambda_{\text {tot }}$ exhibits $6 \%$ pixels more than $\Delta_{\text {co }}$ and $98 \%$ pixels more than $\Delta_{\text {xc }}$. Hence, the difference between $\lambda_{\text {tot }}$ and $\Delta_{\text {co }}$ is significantly reduced with respect to the ascending pass case witnessing that the acquisition geometry plays a key role when observing polarimetric features.

A comparison between ascending and descending results shows that in both cases DP method improves the detection of the damages, see Table 2. However, the improvement is stronger when dealing with the ascending case. It can be also noted that the amount of information carried on co-, i.e.; $\Delta_{\text {co-int }}$, and cross-polarized, i.e.; $\Delta_{\text {xc-int }}$, is different. Although in both ascending and descending cases the copolarized channel contains most of the information, the relative difference between $\Delta_{\text {co-int }}$ and $\Delta_{\text {xc-int }}$ changes from the ascending to the descending passes. In fact, $\Delta_{\text {co-int }}$ results in less detected pixels in the ascending case than the descending case. However, $\Delta_{\mathrm{xc} \text {-int }}$ has more detected pixels in the ascending case than the descending case. This means, that, according to the acquisition geometry, the distribution of information between co- and cross-polarized channels changes.

\section{Conclusions}

In this study, a new paradigm is proposed to detected damages due to the earthquake in DP SAR imagery that is based on the optimisation of the complete covariance matrices collected before and after the seismic event. This paradigm leads to the definition of new features, namely the eigenvalues resulting from the optimisation problem, whose performance is tested considering a meaningful test case, i.e.; the Central Italy earthquake occurred 2016. Experiments, undertaken using Cband Sentinel-1 SAR imagery collected over the city of Amatrice in Lazio (Italy) in ascending and descending mode, confirm the soundness of the proposed approach. The latter is shown to outperform state-of-the-art SP and DP methods in terms of sensitivity to damaged areas. 


\section{References}

Ainsworth, T. L., J. Kelly, J. S. Lee, and J. S. Lee. 2008. "Polarimetric Analysis of Dual Polarimetric SAR Imagery." In 7th European Conference on Synthetic Aperture Radar, 1-4. June.

Albano, M., M. Saroli, M. Moro, E. Falcucci, S. Gori, S. Stramondo, F. Galadini, and S. Barba. 2016. "Minor Shallow Gravitational Component on the Mt. Vettore Surface Ruptures Related to MW 6, 2016 Amatrice Earthquake." Annals of Geophysics 59 (0). doi: $10.4401 /$ ag-7299.

Barboy, B., A. Lomes, and E. Perkalski. 1986. "Cell-Averaging CFAR for Multiple-Target Situations." IEE Proceedings F - Communications, Radar and Signal Processing 133 (2): 176-186. doi: 10.1049/ip-f-1.1986.0028.

Buono, A., F. Nunziata, L. Mascolo, and M. Migliaccio. 2014. "A Multipolarization Analysis of Coastline Extraction Using X-Band COSMO-SkyMed SAR Data." IEEE Journal of Selected Topics in Applied Earth Observations and Remote Sensing 7 (7): 2811-2820. doi: 10.1109/JSTARS.2014.2320366.

Buono, A., F. Nunziata, M. Migliaccio, and X. Li. 2016. "Polarimetric Analysis of CompactPolarimetry SAR Architectures for Sea Oil Slick Observation." IEEE Transactions on Geoscience and Remote Sensing 54 (10): 5862-5874. doi: 10.1109/TGRS.2016.2574561.

Chen, S. W., and M. Sato. 2013. "Tsunami Damage Investigation of Built-Up Areas Using Multitemporal Spaceborne Full Polarimetric SAR Images." IEEE Transactions on Geoscience and Remote Sensing 51 (4): 1985-1997. doi: 10.1109/TGRS.2012.2210050.

Chen, S. W., X. S. Wang, and M. Sato. 2016. "Urban Damage Level Mapping Based on Scattering Mechanism Investigation Using Fully Polarimetric SAR Data for the 3.11 East Japan Earthquake." IEEE Transactions on Geoscience and Remote Sensing 54 (12): 6919-6929. doi: 10.1109/TGRS.2016.2588325.

Cihlar, J., T. J. Pultz, and A. L. Gray. 1992. "Change Detection with Synthetic Aperture Radar." International Journal of Remote Sensing 13 (3): 401-414. doi: 10.1080/01431169208904045.

Cloude, S. 2007. "The Dual Polarisation Entropy / Alpha Decomposition : a Palsar Case Study." In Proceeding in PolInSAR 2007, 1-6. January.

Cloude, S. 2009. Polarisation: Applications in Remote Sensing. OUP Oxford. doi: 10.1093/acprof:oso/9780199569731.001.0001.

Cloude, S. R., and E. Pottier. 1997. "An Entropy Based Classification Scheme for Land Applications of Polarimetric SAR." IEEE Transactions on Geoscience and Remote Sensing 35 (1): 68-78. doi: 10.1109/36.551935.

Ferrentino, E., F. Nunziata, M. Migliaccio, and A. Vicari. 2018. "A Sensitivity Analysis of 
Dual-Polarization Features to Damage Due to the 2016 Central-Italy Earthquake." International Journal of Remote Sensing 0 (0): 1-18. doi: 10.1080/01431161.2018.1466078.

Gamba, P., F. Dell'Acqua, and G. Lisini. 2006. "Change Detection of Multitemporal SAR Data in Urban Areas Combining Feature-Based and Pixel-Based Techniques." IEEE Transactions on Geoscience and Remote Sensing 44 (10): 2820-2827. doi: 10.1109/TGRS.2006.879498.

Gong, L., C. Wang, F. Wu, J. Zhang, H. Zhang, and Q. Li. 2016. "Earthquake-Induced Building Damage Detection with Post-Event Sub-Meter VHR TerraSAR-X Staring Spotlight Imagery." Remote Sensing 8 (11): 887-908. doi: 10.3390/rs8110887.

Hu, H., and Y. Ban. 2014. "Unsupervised Change Detection in Multitemporal SAR Images Over Large Urban Areas." IEEE Journal of Selected Topics in Applied Earth Observations and Remote Sensing 7 (8): 3248-3261. doi: 10.1109/JSTARS.2014.2344017.

Karimzadeh, S., and M. Mastuoka. 2017. "Building Damage Assessment Using Multisensor Dual-Polarized Synthetic Aperture Radar Data for the 2016 M 6.2 Amatrice Earthquake, Italy." Remote Sensing 9 (4). doi: 10.3390/rs9040330.

Lee, J.S., and E. Pottier. 2009. Polarimetric Radar Imaging: From Basics to Applications. Taylor \& Francis. doi: 10.1201/9781420054989.

Marin, C., F. Bovolo, and L. Bruzzone. 2015. "Building Change Detection in Multitemporal Very High Resolution SAR Images." IEEE Transactions on Geoscience and Remote Sensing 53 (5): 2664-2682. doi: 10.1109/TGRS.2014.2363548.

Marino, A., and A. Alonso-Gonzlez. 2017. "An Optimization of the Difference of Covariance Matrices for PolSAR Change Detection." In 2017 IEEE International Geoscience and Remote Sensing Symposium (IGARSS), July.. doi: 10.1109/IGARSS.2017.8128204.

Marino, A., and I. Hajnsek. 2014. "A Change Detector Based on an Optimization With Polarimetric SAR Imagery." IEEE Transactions on Geoscience and Remote Sensing 52 (8): 4781-4798. doi: 10.1109/TGRS.2013.2284510.

Matsuoka, M., and F. Yamazaki. 2004. "Use of Satellite SAR Intensity Imagery for Detecting Building Areas Damaged Due to Earthquakes." Earthquake Spectra 20 (3): 975-994. doi: $10.1193 / 1.1774182$.

Migliaccio, M., F. Nunziata, F. Bruno, and F. Casu. 2007. "Knab Sampling Window for InSAR Data Interpolation." IEEE Geoscience and Remote Sensing Letters 4 (3): 397400. doi: 10.1109/LGRS.2007.895708.

Nunziata, F., M. Migliaccio, and X. Li. 2015. "Sea Oil Slick Observation Using HybridPolarity SAR Architecture." IEEE Journal of Oceanic Engineering 40 (2): 426-440. doi: 10.1109/JOE.2014.2329424.

Park, Sang-Eun, Yoshio Yamaguchi, and Duk jin Kim. 2013. "Polarimetric SAR remote sensing of the 2011 Tohoku earthquake using ALOS/PALSAR." Remote Sensing of 
Environment 132: 212 - 220. doi: 10.1016/j.rse.2013.01.018.

Rignot, E. J. M., and J. J. van Zyl. 1993. "Change Detection Techniques for ERS-1 SAR

Data." IEEE Transactions on Geoscience and Remote Sensing 31 (4): 896-906. doi: $10.1109 / 36.239913$.

Romaniello, Vito, Alessandro Piscini, Christian Bignami, Roberta Anniballe, and Salvatore Stramondo. 2016. "A Multisensor Approach for the 2016 Amatrice Earthquake Damage Assessment." Annals of Geophysics 59 (0). doi: 10.4401/ag-7185.

Sato, M., S. W. Chen, and M. Satake. 2012. "Polarimetric SAR Analysis of Tsunami Damage Following the March 11, 2011 East Japan Earthquake." Proceedings of the IEEE 100 (10): 2861-2875. doi: 10.1109/JPROC.2012.2200649.

Sayama, S., and S. Ishii. 2014. "Suppression of Weather Clutter by Means of LogNormal/CFAR Circuit Modified from CA LOG/CFAR Circuit." Electrical Engineering in Japan 187 (4): 1-9. doi: 10.1002/eej.22514.

Stramondo, S., C. Bignami, M. Chini, N. Pierdicca, and A. Tertulliani. 2006. "Satellite Radar and Optical Remote Sensing for Earthquake Damage Detection: Results from Different Case Studies." International Journal of Remote Sensing 27 (20): 4433-4447. doi: 10.1080/01431160600675895.

Watanabe, M., T. Motohka, Y. Miyagi, C. Yonezawa, and M. Shimada. 2012. "Analysis of Urban Areas Affected by the 2011 Off the Pacific Coast of Tohoku Earthquake and Tsunami With L-Band SAR Full-Polarimetric Mode." IEEE Geoscience and Remote Sensing Letters 9 (3): 472-476. doi: 10.1109/LGRS.2011.2182030.

Watanabe, M., R. B. Thapa, T. Ohsumi, H. Fujiwara, C. Yonezawa, N. Tomii, and S. Suzuki. 2016. "Detection of Damaged Urban Areas Using Interferometric SAR Coherence Change with PALSAR-2." Earth, Planets and Space 68 (1): 131. doi: 10.1186/s40623-016-0513-2.

Yonezawa, C., and S. Takeuchi. 2001. "Decorrelation of SAR Data by Urban Damages Caused by the 1995 Hyogoken-Nanbu Earthquake." International Journal of Remote Sensing 22 (8): 1585-1600. doi: 10.1080/01431160118187. 
Table 1. Sentinel-1 SAR data set

\section{Acquisition date}

Pre-seismic 29 July 2016

Pre-seismic 10 August 2016

Pre-seismic 22 August 2016

Post-seismic 3 September 2016

Pre-seismic 28 July 2016

Pre-seismic 9 August 2016

Pre-seismic 21 August 2016

Post-seismic 2 September 2016

\section{Acquisition pass Polarization}

Ascending

$\mathrm{DP}(\mathrm{VV}+\mathrm{VH})$

Ascending

$\mathrm{DP}(\mathrm{VV}+\mathrm{VH})$

Ascending

$\mathrm{DP}(\mathrm{VV}+\mathrm{VH})$

Ascending

$\mathrm{DP}(\mathrm{VV}+\mathrm{VH})$

Descending

$\mathrm{DP}(\mathrm{VV}+\mathrm{VH})$

Descending

$\mathrm{DP}(\mathrm{VV}+\mathrm{VH})$

Descending

$\mathrm{DP}(\mathrm{VV}+\mathrm{VH})$

Descending

Table 2. CA-CFAR detection performance for the whole data set

\section{Multi-polarization features}

\section{Detected} pixels
Acquisition pass

Ascending

Ascending

Ascending

Descending

Descending

Descending 


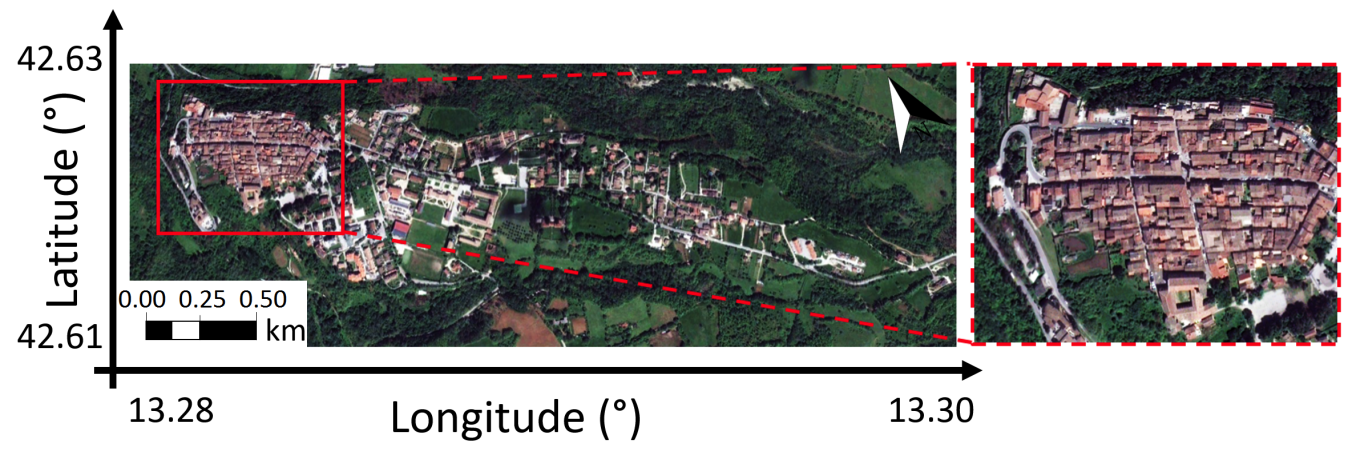

(a)

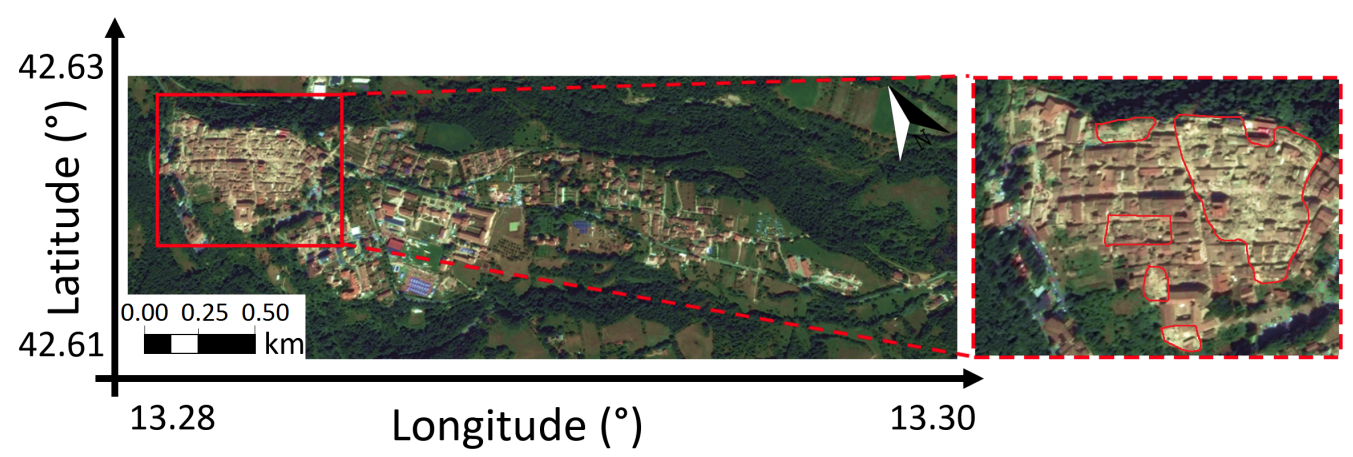

(b)

Figure 1. Google Earth(c) images related to the city of Amatrice and collected before $(a)$ the seismic event (on 21 May 2016) and after (b) the seismic event (on 25 August 2016). On the right hand side of the images an enlarged version of the area enclosed by the red box is shown. This is the area that experienced major damages, which are enclosed by red polygons. 


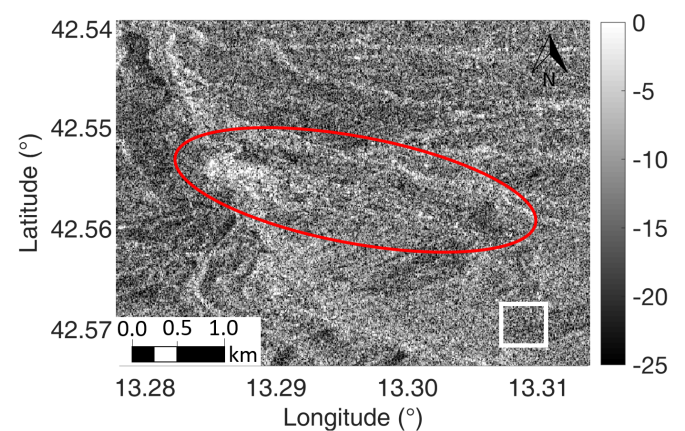

(a)

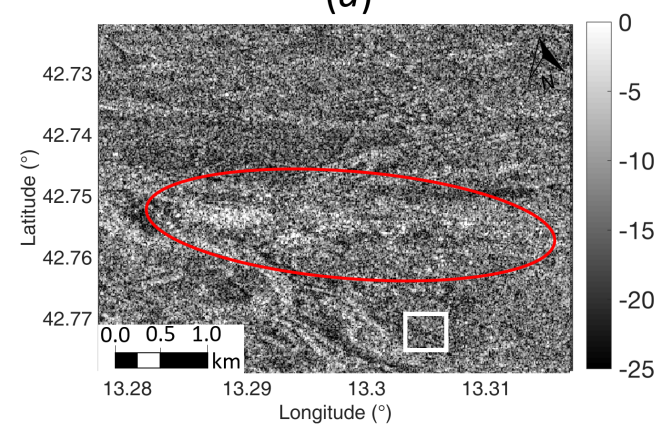

(c)

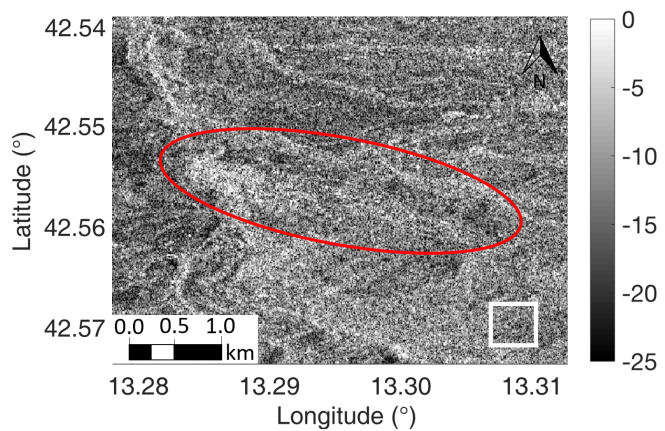

(b)

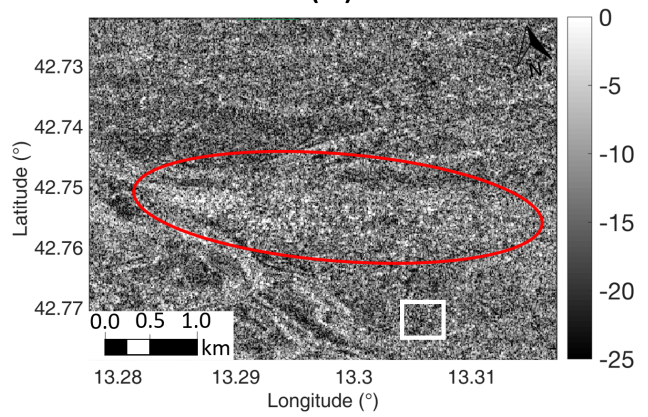

(d)

Figure 2. Excerpt of Sentinel-1 ground-projected VV-polarized SAR imagery collected over the area of Amatrice, Italy. The figure is organized in matrix format: the first and the second rows stand for SAR imagery collected in ascending and descending mode, respectively. The first and the second columns refer to SAR imagery acquired before and after the earthquake. respectively. 


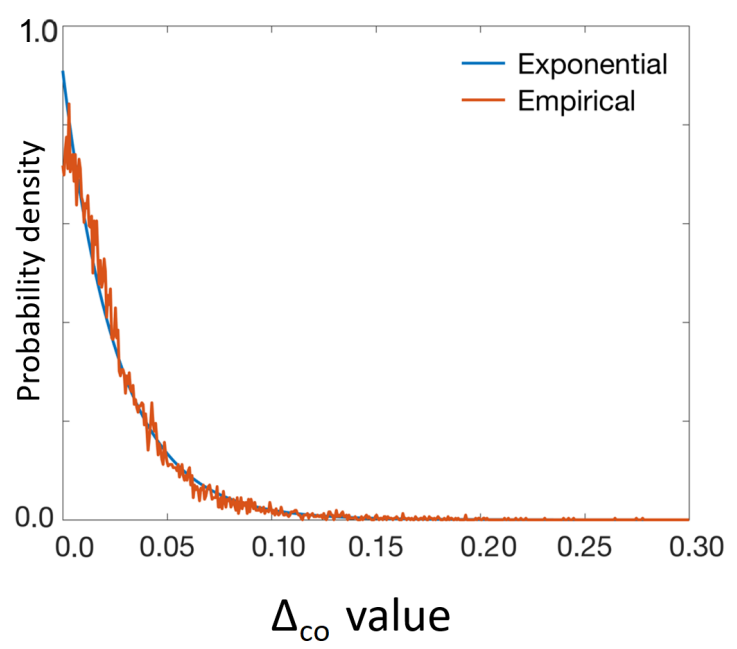

(a)

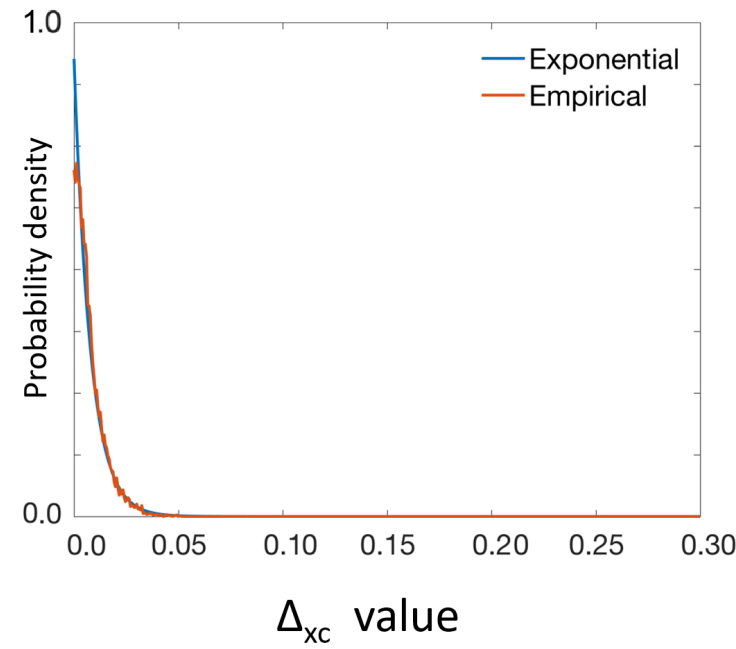

(b)

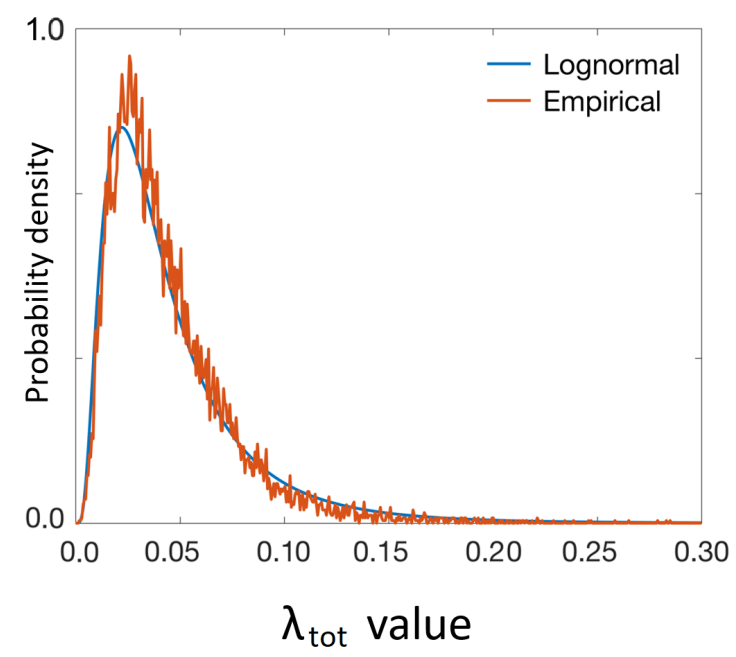

(c)

Figure 3. Empirical pdfs related to $\Delta_{\text {co }}(a), \Delta_{\mathrm{xc}}(b)$ and $\lambda_{\text {tot }}(c)$ evaluated within a background clutter ROI. Note that, for reference purposes, theoretical exponential ( $a$ and $b$ ) and Lognormal $(c)$ pdfs are also annotated in blue. 


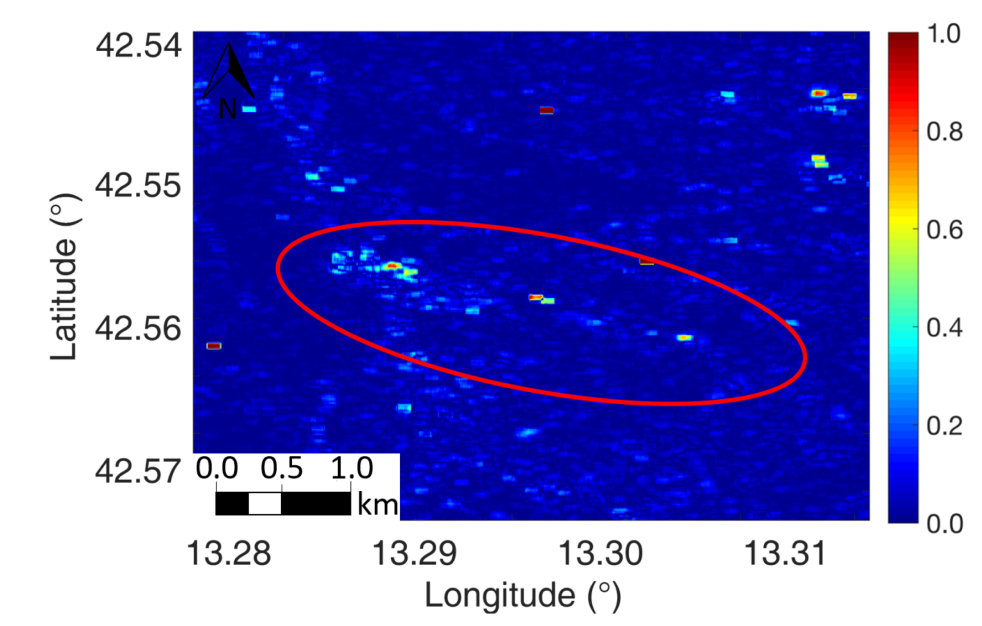

(a)

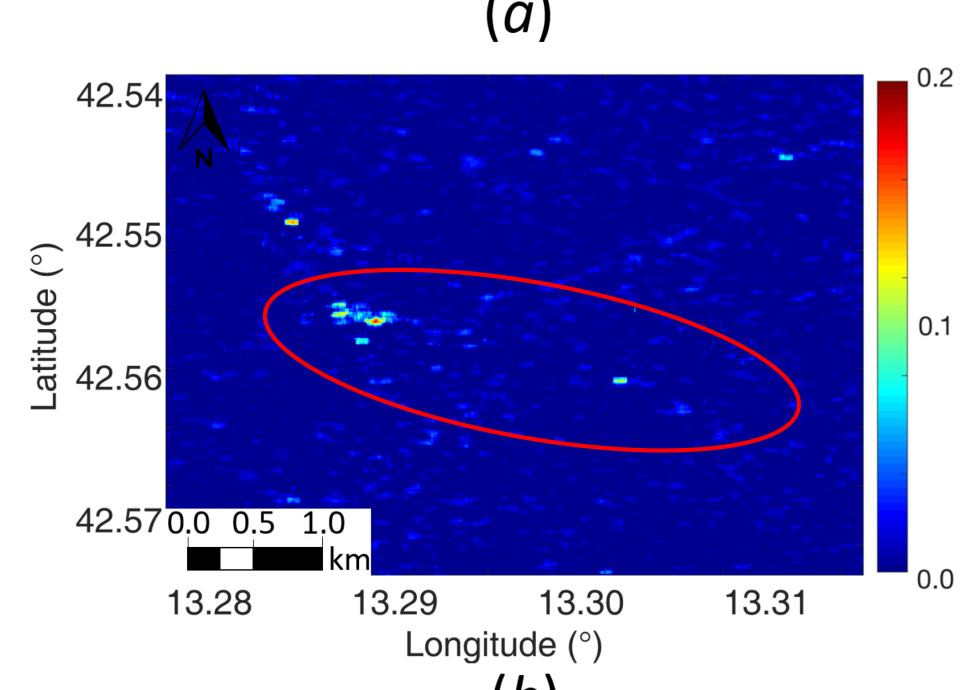

(b)

Figure 4. $\Delta_{\mathrm{co}}(a)$ and $\Delta_{\mathrm{xc}}(b)$ false colour imagery that refer to an excerpt of the SAR scenes collected

in ascending mode (see Table 1) where the urban area of Amatrice is circled in red. 


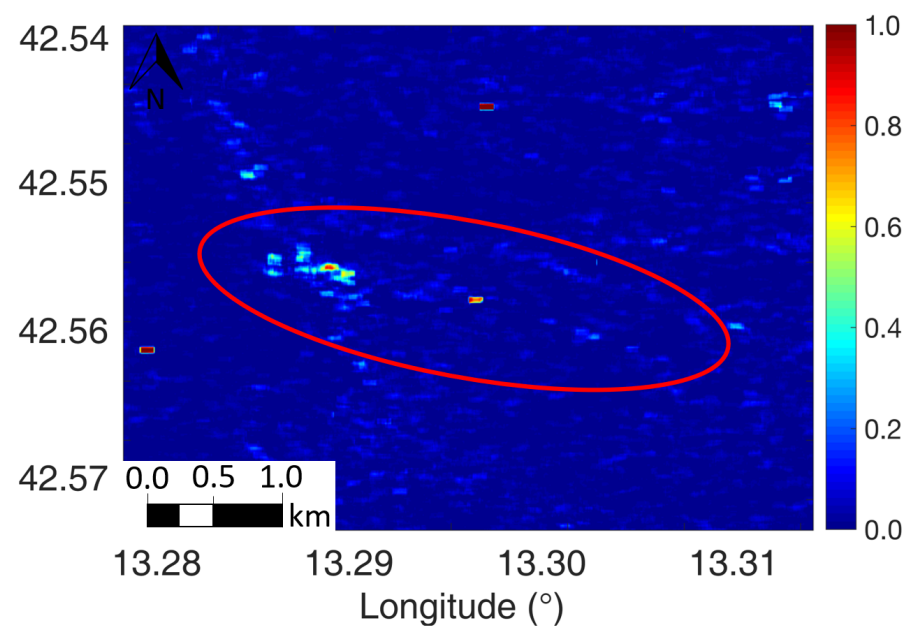

(a)

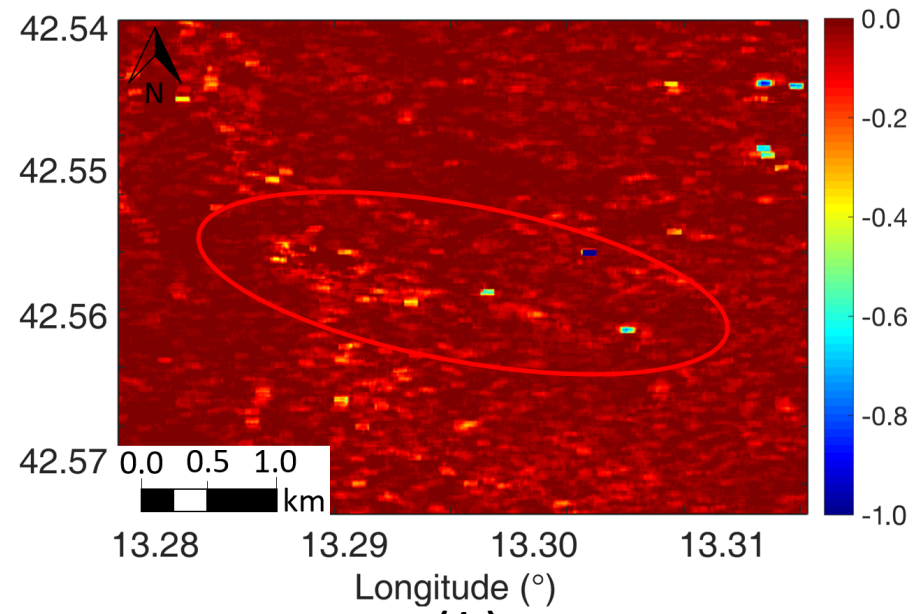

(b)

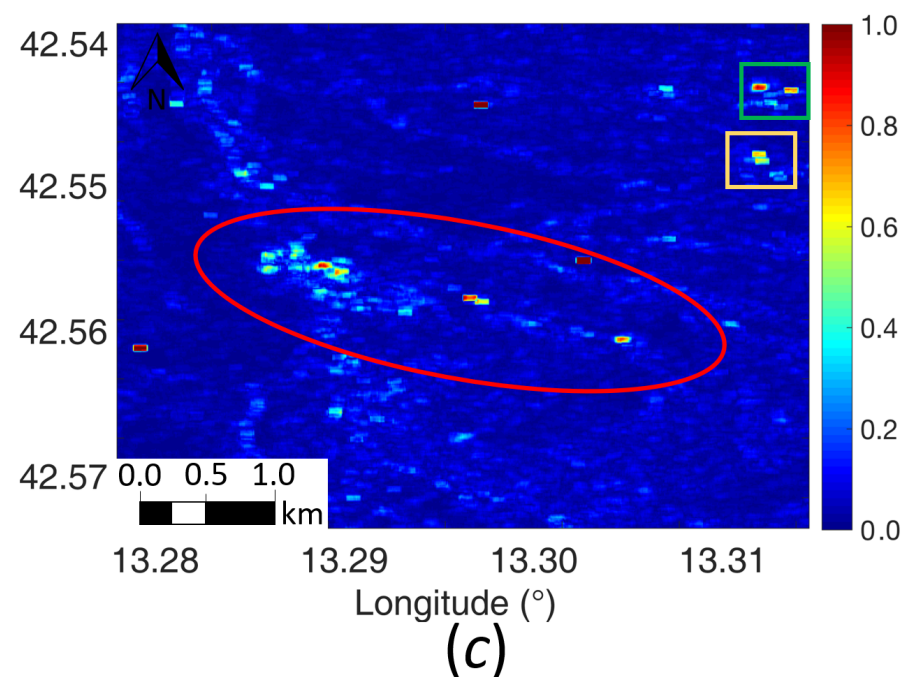

Figure 5. $\lambda_{1}(a), \lambda_{2}(b)$ and $\lambda_{\text {tot }}(c)$ false colour imagery that refer to an excerpt of the SAR scenes collected in ascending mode (see Table 1) where the urban area of Amatrice is circled in red. 


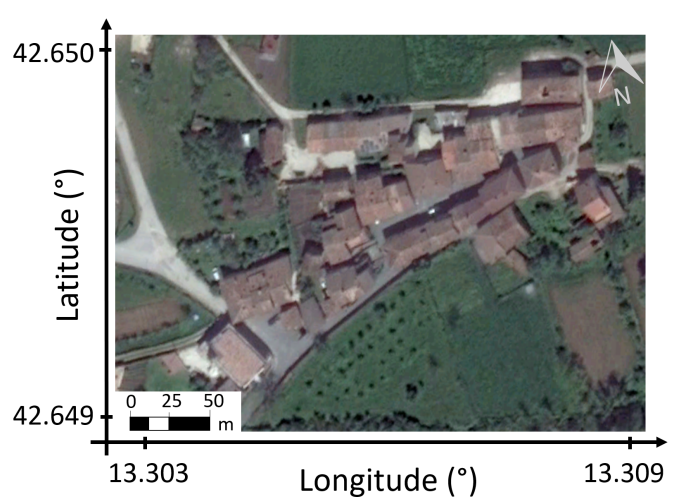

(a)

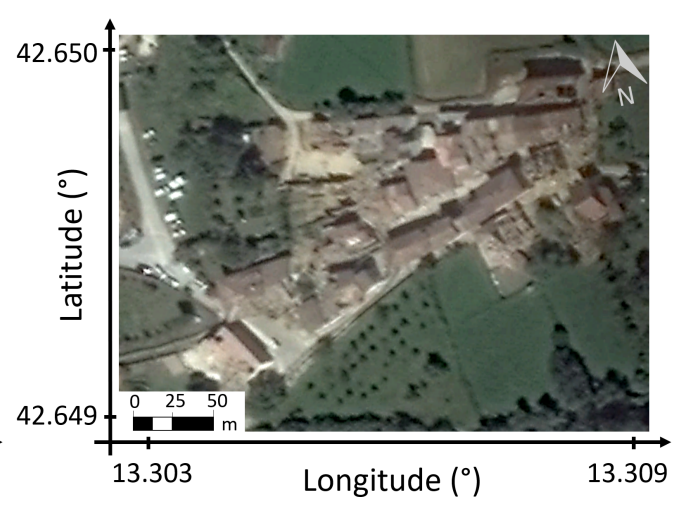

(b)

Figure 6. Google Earth (C) images related to the city of Castel Sant'Angelo and collected before (a) the seismic event (on 21 May 2016) and after (b) the seismic event (on 25 August 2016)

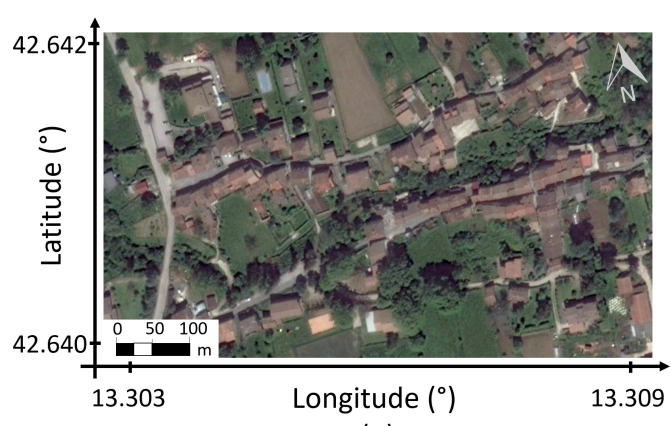

(a)

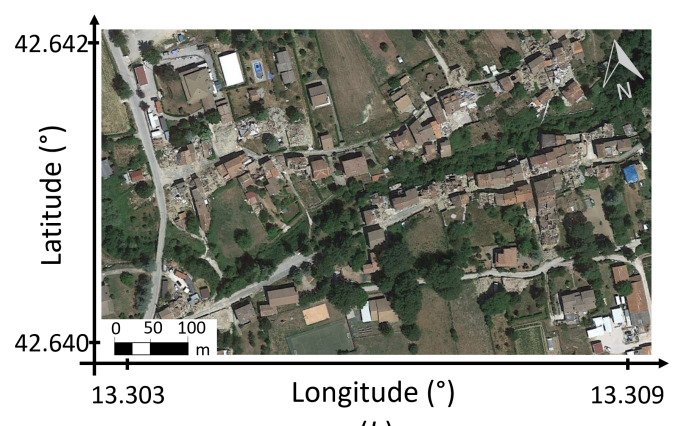

(b)

Figure 7. Google Earth ( ) images related to the city of Sommati and collected before $(a)$ the seismic event (on 21 May 2016) and after (b) the seismic event (on 7 July 2017)

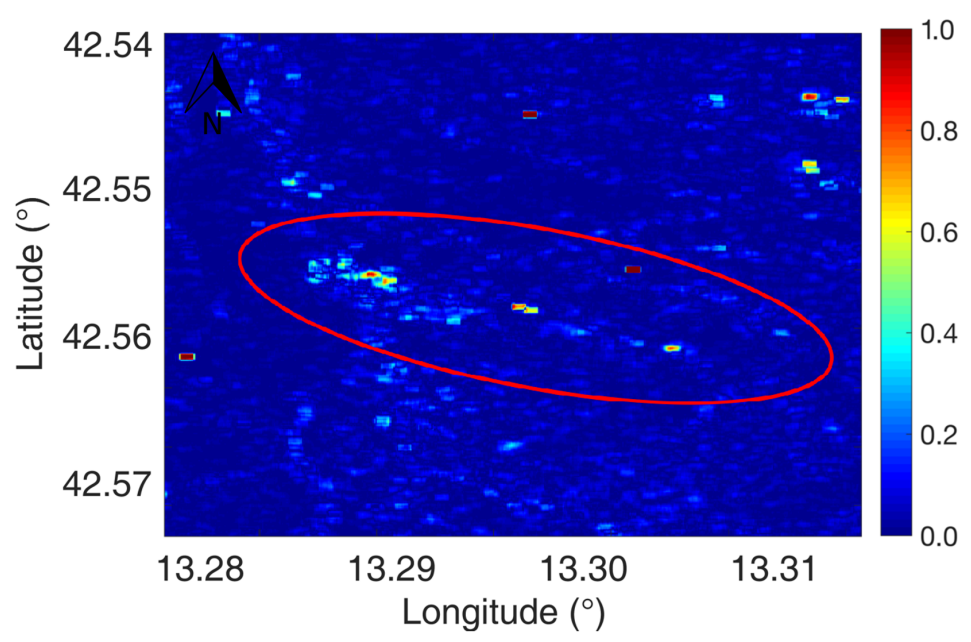

Figure 8. SPAN false colour image that refer to an excerpt of the SAR scenes collected in ascending mode (see Table 1) where the urban area of Amatrice is circled in red. 


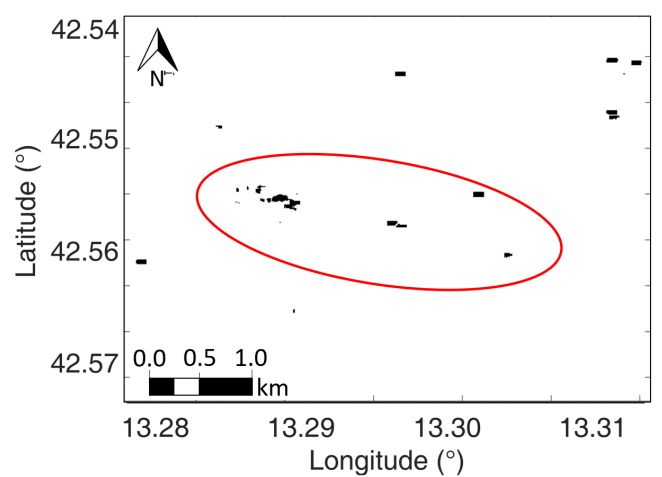

(a)

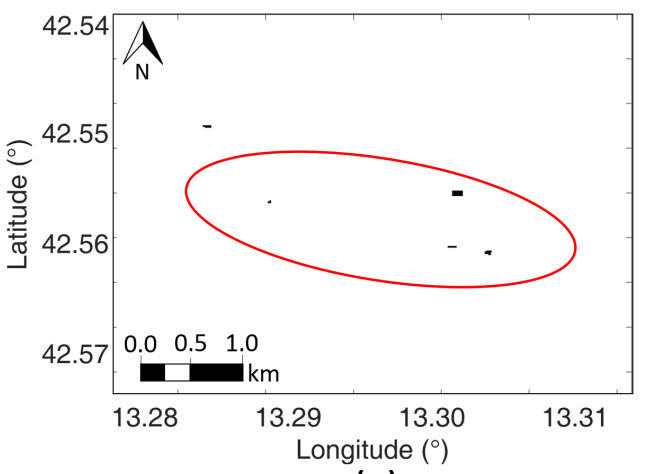

(c)

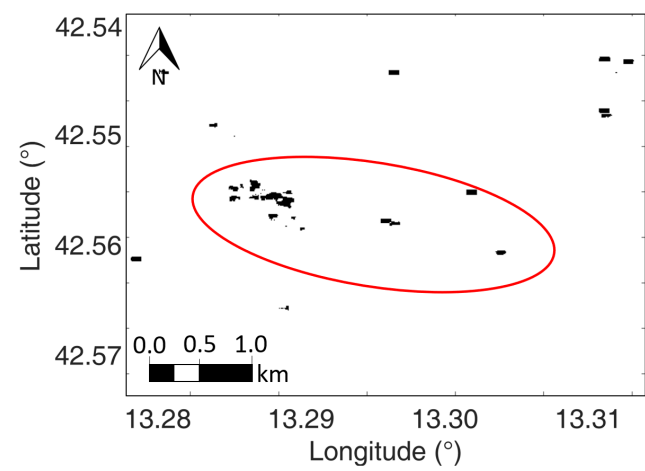

(e)

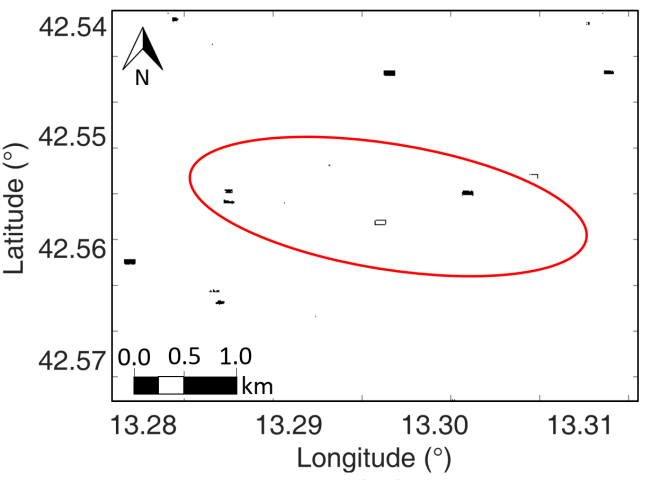

(b)

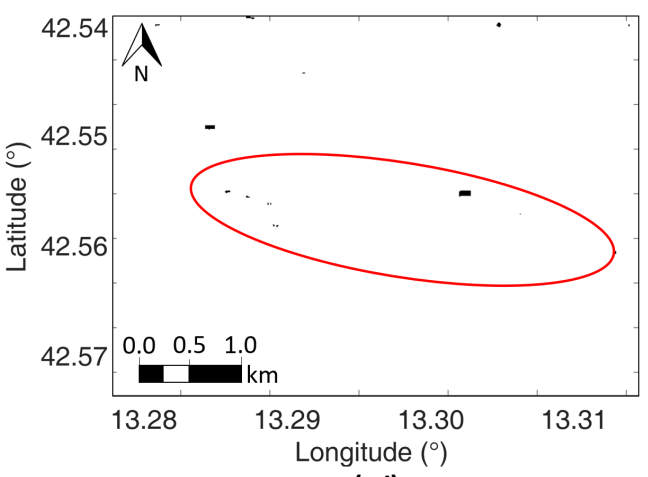

(d)

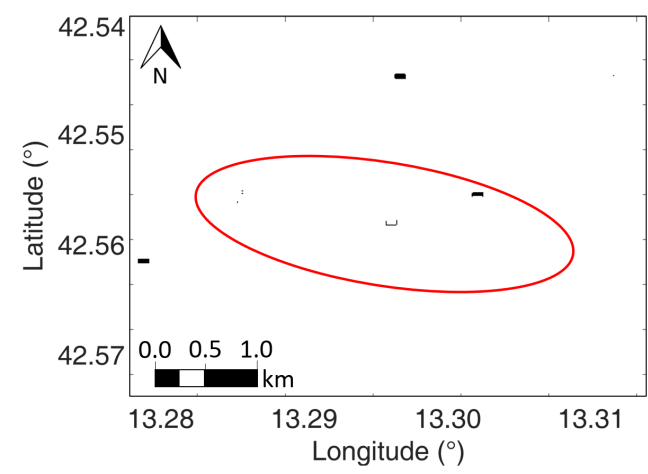

$(f)$

Figure 9. Binary imagery obtained applying CA-CFAR method with a $P_{f a}$ equal to $10^{-5}$ to the imagery of Figure 4 and 5 The figure is organized in matrix format: the first column refers to the inter-seismic case; while the second column refers to the pre-event case. The three rows refer to $\Delta_{\mathrm{co}}, \Delta_{\mathrm{xc}}$ and $\lambda_{\text {tot }}$, respectively. 

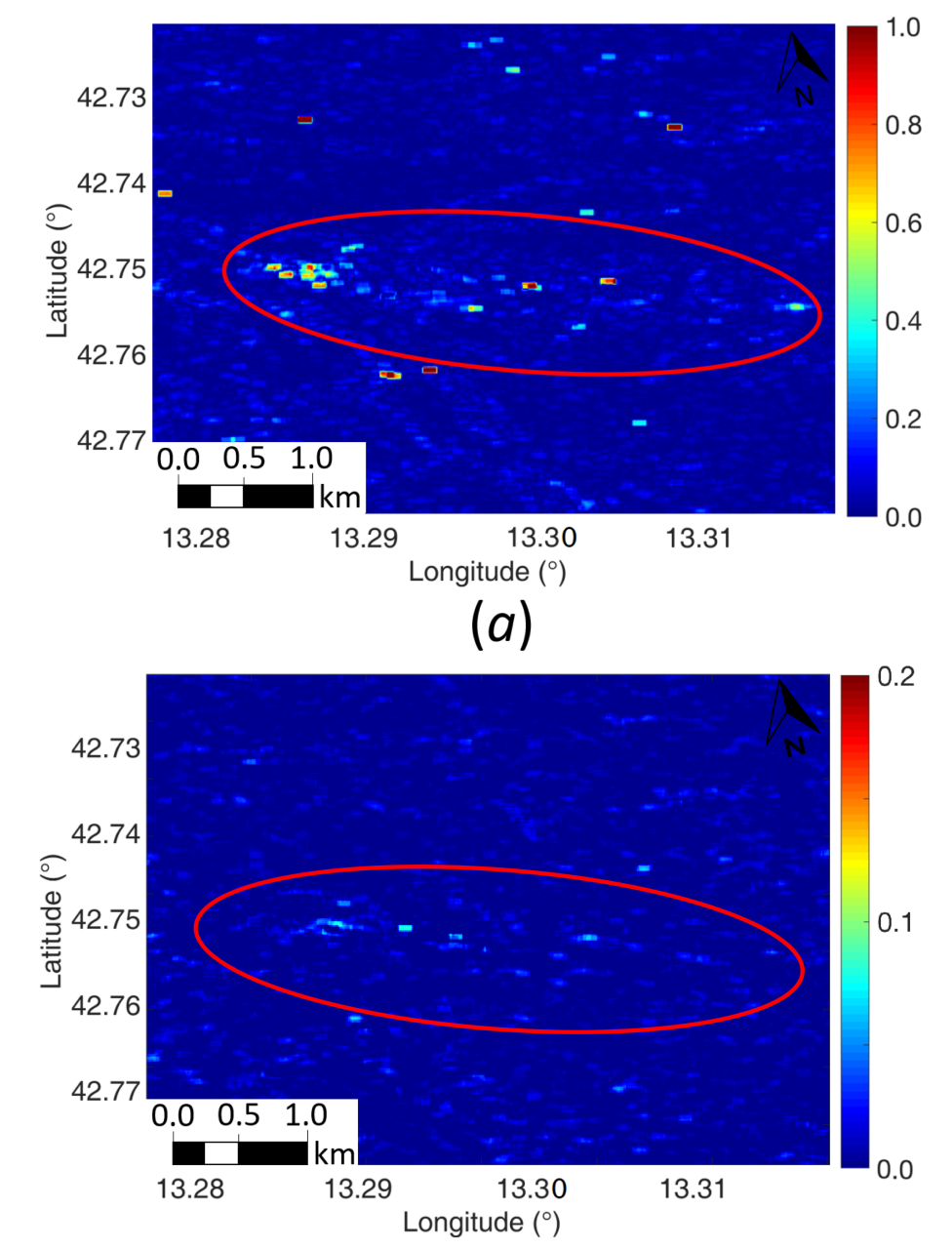

(b)

Figure 10. $\Delta_{\text {co }}(a)$ and $\Delta_{\text {xc }}(b)$ false colour imagery that refer to an excerpt of the SAR scenes collected in descending mode (see Table 1 where the urban area of Amatrice is circled in red. 

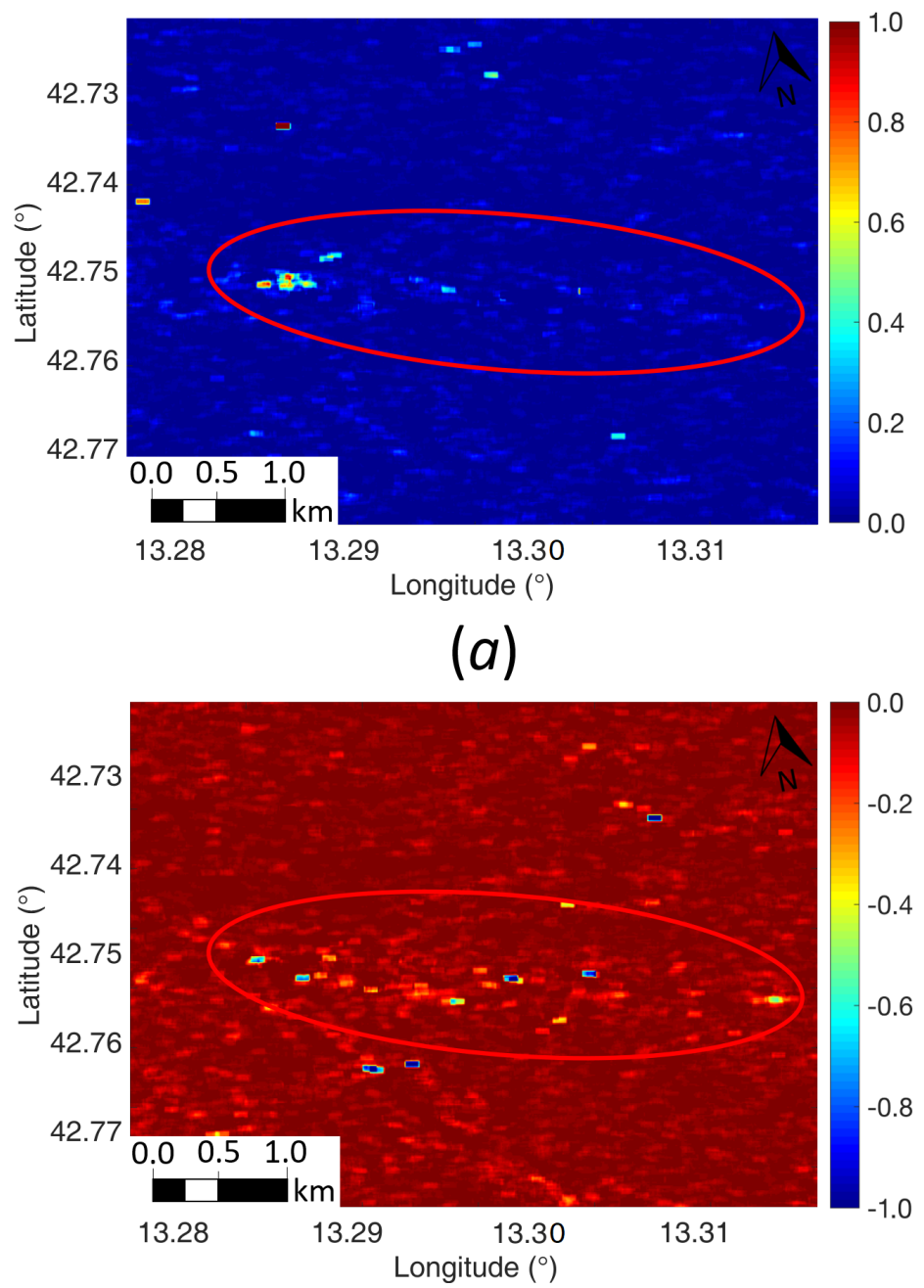

(b)

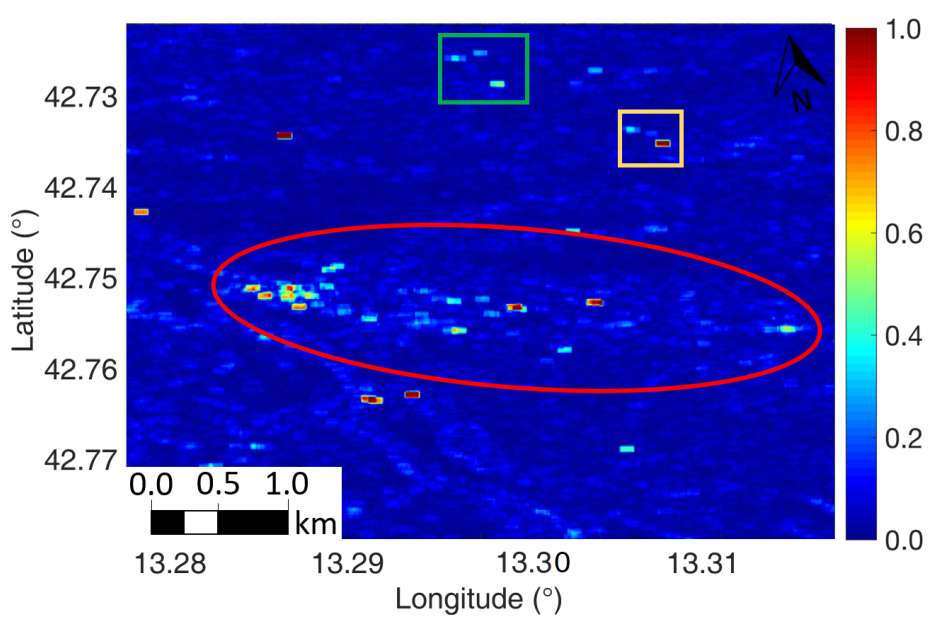

(c)

Figure 11. $\lambda_{1}(a), \lambda_{2}(b)$ and $\lambda_{\text {tot }}(c)$ false colour imagery that refer to an excerpt of the SAR scenes collected in descending mode (see Table 1) where the urban area of Amatrice is circled in red. 


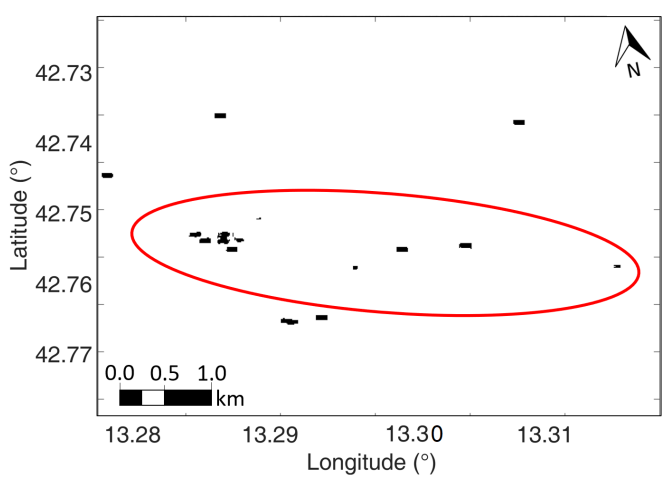

(a)

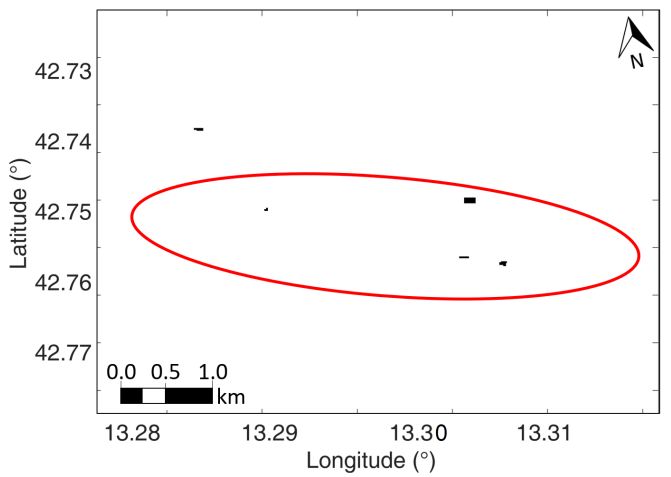

(c)

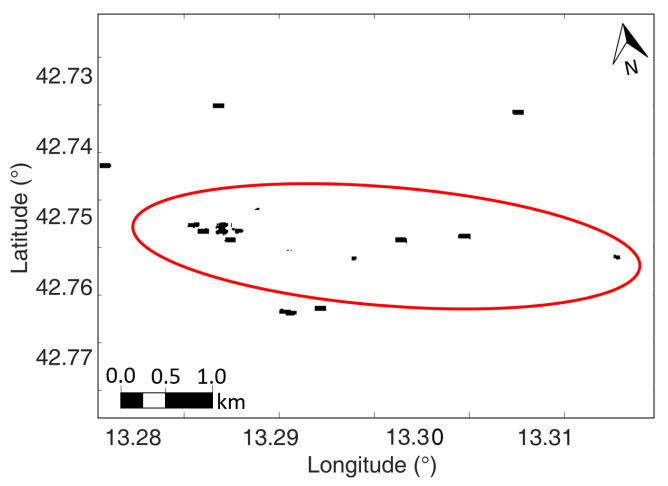

(e)

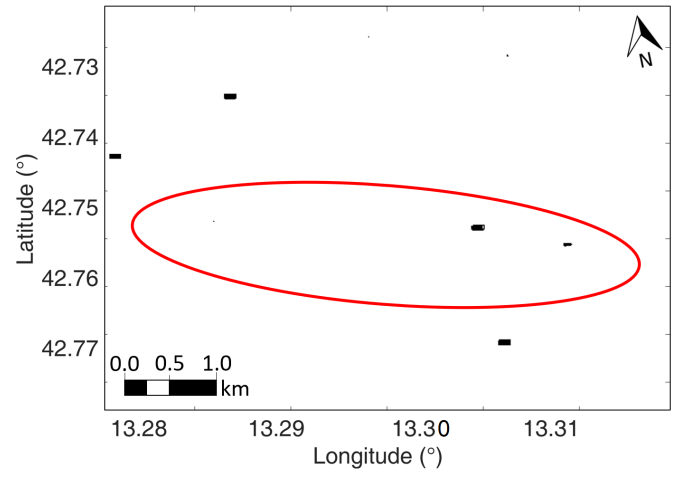

(b)

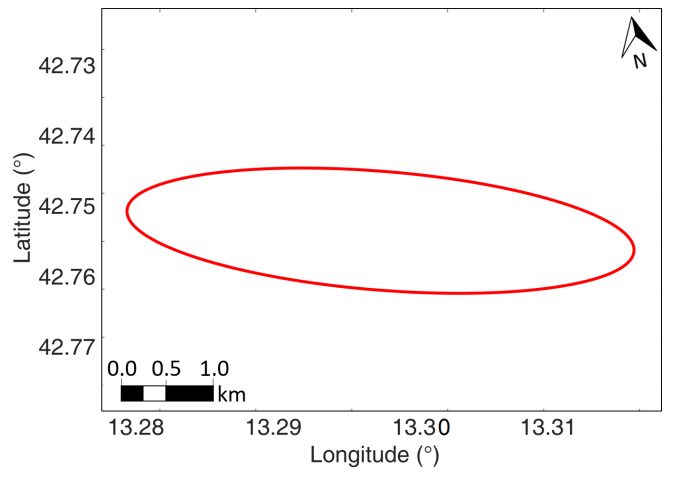

(d)

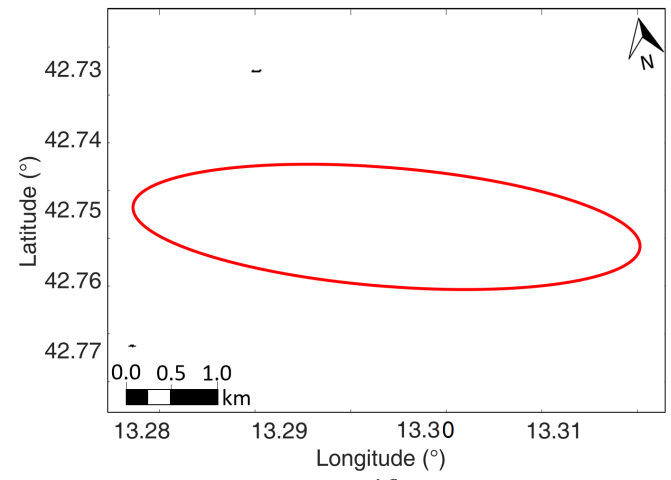

(f)

Figure 12. Binary imagery obtained applying CA-CFAR method with a $P_{f a}$ equal to $10^{-5}$ to the imagery of Figure 10 and 11 The figure is organized in matrix format: the first column refers to the inter-seismic case; while the second column refers to the pre-event case. The three rows refer to $\Delta_{\mathrm{co}}, \Delta_{\mathrm{xc}}$ and $\lambda_{\text {tot }}$, respectively. 